\title{
FASCINATING OPTICS \\ IN A GLASS OF WATER
}

\author{
iby A.T.A.M. de Waele \\ I Dotterbeek 8, NL5501BH - Veldhoven, The Netherlands - DOI: http://dx.doi.org/10.1051/epn/2016205 \\ In Ref. [1] Jo Hermans discussed how the image of a horizontal arrow behind a glass of water reverses direction \\ when it is moved away from the glass. The present paper deals with some other interesting effects that can \\ be observed when looking at a drawing (source) taped tightly onto the back of a cylindrical glass of water, or \\ beer, or white wine, whatever the reader prefers.
}

v FIG. 1: (a) Cartoon, obtained from Ref.

1 , which is used as source in Figs. $b$ and $c$.

(b) Photo taken

through a plexiglass cylinder with the focus of the camera on the upper rim of the glass of beer. (c) Photo with the focus on the left vertical edge of the glass of beer n order to introduce the topic we take the cartoon from Ref. [1] (Fig. 1a) and tape it onto the back of a glass of water. If we try to take a photo of the cartoon with a camera in autofocus mode it has problems finding the focus. It is possible to focus the camera manually on the horizontal lines in the cartoon, e.g., the upper rim of the beer glass (Fig. 1b) but then the vertical lines are blurred. If the focus is set on the vertical lines (Fig. 1c) all horizontal lines are blurred.

Another interesting effect is observed if a cotton wire is taped vertically onto a glass half-filled with water as in Fig. 2. If the wire is near the center, one image is seen through the water. If the glass is rotated clockwise the wire and its image both move to the right, but the image moves faster than the wire. At some point, surprisingly, a second image jumps in at the right edge of the glass. If the glass is rotated further, the left image moves to the right and the right image moves to the left. At some angle the two images coincide. If the glass is rotated still further both images disappear: the wire is invisible through the water. Also the focal properties of the images, especially in the position where the two images tend to coincide, are surprising, as will be discussed below.

Fig. 3 shows the image of a grid of straight lines, taped onto the glass. It is seen to consist of a series of strongly

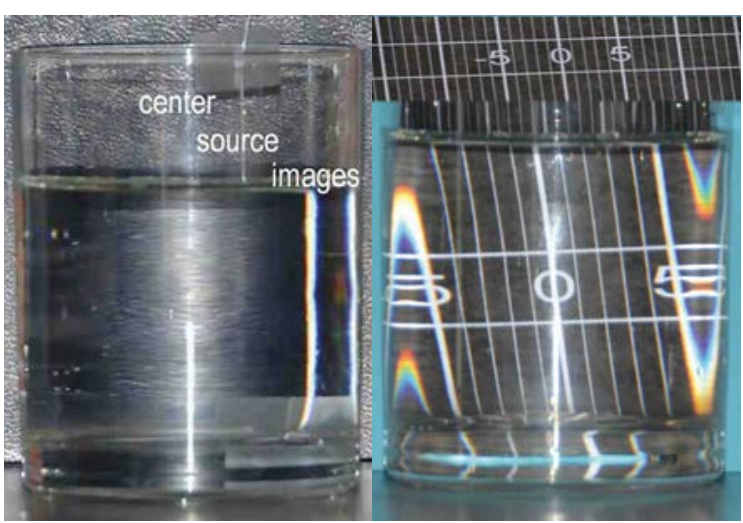

$\Delta$ FIG. 2: Picture of a cotton thread vertically taped onto the back of a glass partially filled with water. The middle of the glass is shown by the line of reflection of the flash light. The cotton thread (source) can be seen through the glass above the water level. The two images can be seen on the right.

$\Delta$ FIG. 3: Top: Slightly tilted grid of white straight lines on a black background. Bottom: Picture of the grid through the water.

bent curves with sharp maxima and minima. Near the extrema beautiful rainbow spectra are observed.

All phenomena described in this paper can be understood by using simple geometrical optics. The experiments can be repeated with simple means and observed with the naked eye and/or a camera with manual focus.

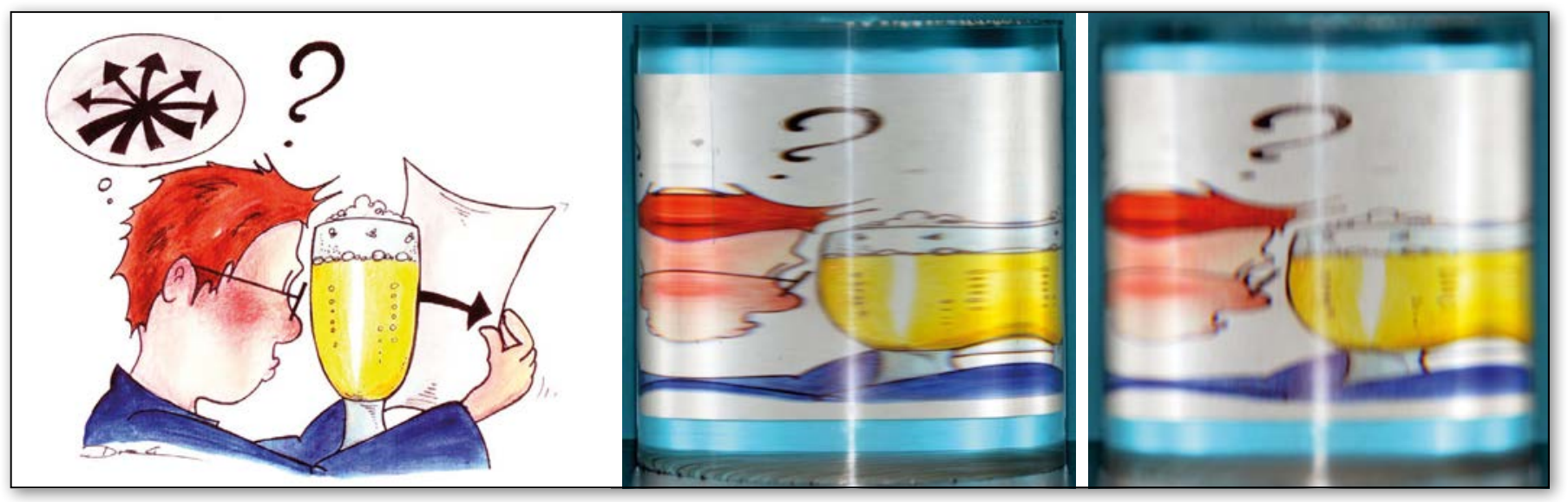




\section{The position of the image}

The perception of depth has several definitions [2],[3]. One is the binocular depth perception where the position of the image is determined by the point of intersection of one light ray reaching the right eye and the other ray reaching the left eye. In the monocular depth perception the position of the observation point is varied only over the diameter of the pupil of the eye (a few $\mathrm{mm}$ ). This is also used by the eye to observe an image as sharp.

The system under consideration is essentially three dimensional, even if the source and the observer are in one horizontal plane. Two light rays in the vertical plane are deflected by a straight line (Fig. 4) while two rays in the horizontal plane are deflected by a circle (Fig. 5). As a result the positions of the images, constructed with rays in the vertical plane or in the horizontal plane, differ. This difference is a well-known property of astigmatic lenses ([3], page 162). In fact, it is used as a test for astigmatism.

\section{Rays in the vertical plane}

First we calculate the position $x_{I}$ of the image of a source (object) at $x_{S}$ in a medium with index of refraction $n$ and a flat surface (perpendicular to the $\mathrm{x}$-axis) to air at $x_{B}$. This corresponds with light rays in the vertical plane of our problem. Using Fig. 4 we find

$$
h=\left(x_{B}-x_{S}\right) \tan \varphi=\left(x_{B}-x_{I}\right) \tan \alpha .
$$

where points left from $\mathrm{O}$ are taken negative, $\varphi$ the angle of incidence and $\alpha$ the angle of refraction. Here $x_{B}-x_{S}$ is the distance between the source and the surface and $x_{B}-x_{I}$ the distance between the image and the surface. Snell's law of refraction reads

$$
\sin \alpha=n \sin \varphi .
$$

For small angles $(\alpha, \varphi \ll 1)$ Eqs. (1) and (2) reduce to

and

$$
\left(x_{B}-x_{S}\right) \varphi=\left(x_{B}-x_{I}\right) \alpha
$$

$$
\left(x_{B}-x_{S}\right)=\left(x_{B}-x_{I}\right) n .
$$

For $n \approx 4 / 3$ (water) we see that a diver or snorkeler, looking at the sea through his diving mask, observes fish $25 \%$ closer than they really are.

In our case the source is taped onto the back of a cylinder. With the origin at the axis $x_{B}=-x_{S}$ (see Fig. 5) and Eq. (4) with $n \approx 4 / 3$ yields $x_{I} \approx 0.50 x_{S}$. As $x_{S}$ and $x_{I}$ are both negative the image is halfway between the origin and the back of the glass.

With a camera focused on this $x_{I}$ the image of a point source is a small horizontal line. If the source is a horizontal wire, taped onto the cylinder of radius $R_{C}, x_{S}$ varies from $-R_{C}$ to 0 so, in principle, $x_{I}$ varies from $-0.50 R_{C}$ to 0 . However, as we will see later, only $23.5 \%$ from the back is visible so $x_{I}$ is always close to $-0.5 R_{C}$. Practically speaking, horizontal lines can be observed as sharp over the whole visible range of the source.

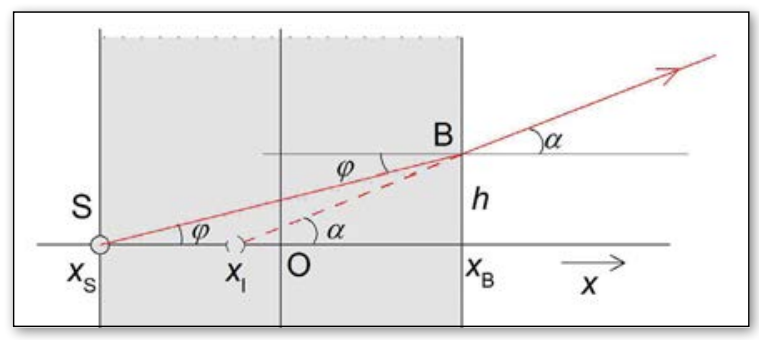

4 FIG. 4: Image formation by rays in the vertical plane by flat surfaces.

The positions of $x_{S}$ and $x_{B}$ in the case of a cylinder are indicated in FIG. 5.

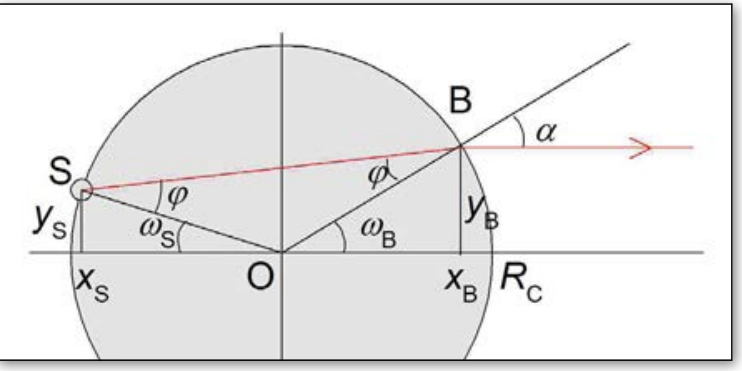

4 FIG. 5: Image formation by rays in the horizontal plane, with the observer at infinity.

\section{Rays in the horizontal plane}

Next we consider the image formation in the horizontal plane. This corresponds to determining the position of the image of a vertical wire. The situation in which the observer is at infinity is depicted in Fig. 5. The angle of incidence $\varphi$ is the angle between SB and the normal to the surface at B (i.e., OB). Since $\omega_{S}+\omega_{B}=2 \varphi$ and $\alpha=\omega_{B}$, Eq. (2) reads

$$
\text { So } \begin{aligned}
& \sin \omega_{B}=\operatorname{nsin}\left(\left(\omega_{S}+\omega_{B}\right) / 2\right) . \\
& \omega_{S}=2 \arcsin \left(\left(\sin \omega_{B}\right) / n\right)-\omega_{B} .
\end{aligned}
$$

This relation is plotted in Fig. 6. For $\omega_{B}=0$ we see that also $\omega_{S}=0$, so source and image coincide. Rotating the cylinder clockwise means increasing $\omega_{S}$. Initially $\omega_{S}$ and $\omega_{B}$ both increase. However, at $\omega_{B}=\omega_{B 2}, \omega_{S}$ reaches a maximum $\omega_{S 2}$. From now on increasing $\omega_{B}$ decreases $\omega_{s}$. For an image at the edge of the cylinder $\left(\omega_{B}=\pi / 2\right)$ the source is at $\omega_{S 1}$. For $0<\left|\omega_{S}\right|$ $<\omega_{S 1}$ there is one image, for $\omega_{S 1}<\left|\omega_{S}\right|<\omega_{S 2}$ there are two solutions for $\omega_{B}$ so one source has two images. At $\left|\omega_{S}\right|=\omega_{S 2}$ the two images coincide. For $\left|\omega_{S}\right|>\omega_{S 2}$ there is no image: a wire in this region is invisible. For $\mathrm{n}=1.33$ we have $\omega_{B 2}=1.040 \mathrm{rad}$, $\omega_{S 1}=0.131 \mathrm{rad}$, and $\omega_{S 2}=0.371 \mathrm{rad}$. So less than one quarter $(0.371 /(\pi / 2)=0.236)$ of the back is visible through the glass.

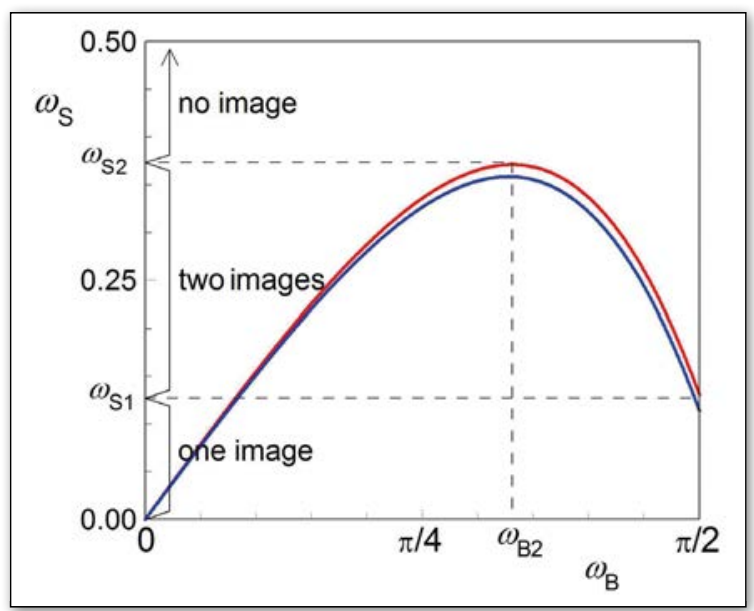

4FIG. 6: $\omega_{\mathrm{s}}$ as function of $\omega_{B}$ (Eq. (6)) for water for $n=1.33$ (red light) and $n=1.34$ (violet light). The $\omega_{s}$-regions with one image, two images, and no image are indicated. 


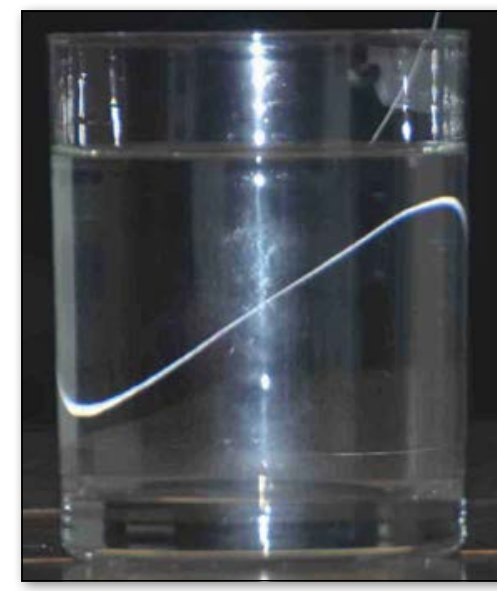

4 FIG. 7: Picture

of a tilted wire

observed through

a glass of water.

The right-hand

side of the curve

is quite similar to

the curve in FIG.6.

In Fig. 6 a curve is also drawn for violet light $(n=1.34)$. In this case $\omega_{s 2}=0.358 \mathrm{rad}$. A variation of $n$ of $0.75 \%$ leads to a variation of $\omega_{S 2}$ of $3.6 \%$. So the values of the extrema are rather strong functions of $n$ which explains the rainbow phenomena in Fig. 3.

\section{Tilted wire}

Instead of rotating the glass with a vertical wire we can also tape a slightly tilted wire onto the back of the glass. The vertical position is proportional to $\omega_{S}$ so we see the whole sequence of events in one glance (Fig. 7). The horizontal position is not proportional to $\omega_{B}$ but to $\sin \omega_{B}$, so the image is a somewhat distorted version of the curve of Fig. 6 but it shows the same features.

\section{The $\mathrm{x}$-positions of the images}

The manual focus of a camera can be used to obtain a sharp picture of the vertical images as in Fig. 2. It turns out that the focus of the right image is in front of the glass, whereas the focus of the left image is behind it. If $\omega_{S}=$ $\omega_{S 2}$, where the two images coincide, one image is at $x_{I}=$ $-\infty$ and the other at $+\infty$. This spectacular discontinuity is demonstrated in Fig. 8. The source is a slightly-tilted grating of yellow lines. In the left picture the focus of the camera is set in front of the glass and in the right
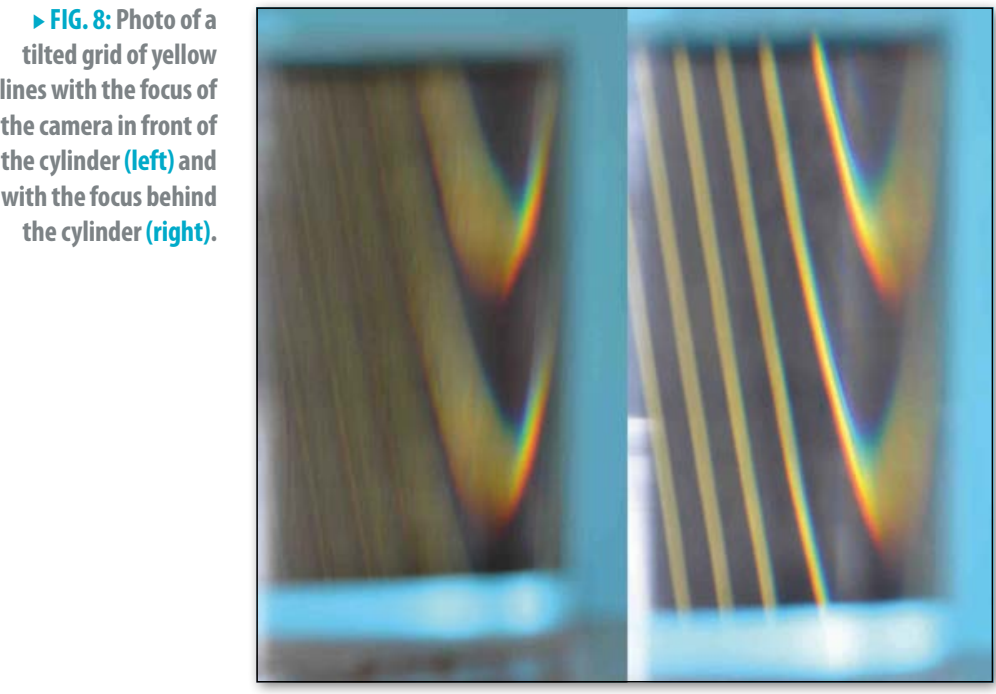

picture far behind it. In both pictures the sharpness of the images has a pronounced discontinuity at the minima of the curves.

If we use a point source at a position that gives two images $\left(\omega_{S 1}<\left|\omega_{S}\right|<\omega_{S 2}\right)$ and increase the focal distance of the camera manually from zero to infinity we see the following sequence (apart from blurred images): first we see a sharp small vertical line segment on the right, next we see two small sharp horizontal lines, and finally we see one small sharp vertical line at the left. These phenomena can be understood using relatively simple geometrical optics but this is beyond the scope of this paper.

\section{Discussion}

The focus of rays in the horizontal plane is located inside the cylinder, about half a radius behind the cylinder axis and does not vary very much. By contrast, the focus of rays in the vertical plane is a strong function of the position of the source. In general, the distance between the focal planes of horizontal and vertical lines is typically a few times the diameter of the cylinder.As a result, a camera cannot take a photo in which all lines are sharp. Surprisingly enough this confusing situation does not result in a blurred image if the source is observed with the naked eye: one sees a perfectly sharp image even in monocular vision. For example, the pixels in the cheek of the cartoon of Fig. la can be seen very clearly. Apparently the human perception can handle this confusing information and construct a sharp image. How it manages to do that is a very interesting topic, but outside the scope of this paper.

Another interesting observation is the following: the drawing of Fig. 1a is taped onto the back of the glass, so it is evidently curved. However, if one looks at the cartoon through the glass of water the image is rather flat. Perhaps this is related to the fact that only the central $25 \%$ of the cartoon is visible, but it may also have to do with the way our eyes and brain handle the optical information.

\section{About the author}

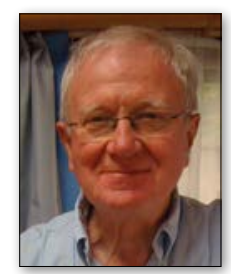

Alphons (Fons) de Waele is emeritus professor of physics at Eindhoven University of Technology. His main interests are thermodynamics and, in particular, refrigeration technology. He has been director of education and has written several articles in Wikipedia.

\section{References}

[1] L.J.F. Hermans, Europhysics News 44/5, 15 (2013);

[2] D.S. Falk, D.R. Brill, and D.G. Stork, Seeing the light, Optics in Nature, Photography, Color, Vision, and Holography (1986, Harper\&Row, Publishers, New York)

[3] D. Rogers-Ramachandran and V.S. Ramachandran, Scientific American, July 1, 2009

[4] http://refractiveindex.info/ 